\title{
Clinical Profile of Patients with Hip Hemi-arthroplasty Managed at the Physiotherapy Department of a Nigerian Tertiary Health Institution between 2008 and 2013
}

\author{
Odusanya $\mathrm{OF}^{1}$, Odole $\mathrm{AC}^{2}$ \\ ${ }^{1}$ Department of Physiotherapy, University College Hospital, Ibadan, Oyo State, Nigeria \\ ${ }^{2}$ Department of Physiotherapy, College of Medicine, University of Ibadan, Ibadan, Oyo State, Nigeria
}

\begin{abstract}
Correspondence
Mr OF Odusanya, Department of Physiotherapy, University College Hospital, Ibadan, Oyo State, Nigeria • oyinlolafo@yahoo.com
\end{abstract}

\begin{abstract}
SUMMARY
This study was a review of individuals who had hip hemi-arthroplasty and were managed at the physiotherapy department of a Nigerian tertiary health institution. Information on age, sex, causes of fracture, types of referral, outcomes assessed, treatment provided and discharge pattern were obtained from physiotherapy patients' register, case files and nursing registers. Data was summarized using descriptive statistics of range, mean, standard deviation, and percentages. A total of 57 cases (26 males; 31 females) aged $76.77 \pm 11.59$ years (32-92 years) was reviewed. The majority $(98.2 \%)$ had hip hemi-arthroplasty secondary to femoral neck fracture. Only one case was due to femoral head damage secondary to sickle cell disease. The cause of fracture was documented in 21 cases with $76 \%$ of the fractures resulting after falls. Forty-one patients $(71 \%)$ were referred for ambulation only post-surgery while sixteen $(29 \%)$ were referred for full physiotherapy. Pain and muscle strength were the only impairment variables assessed in those referred for physiotherapy. Fifty-five $(91 \%)$ were discharged home with walking aids while three $(6 \%)$ were deceased. Fourteen $(25 \%)$ continued physiotherapy on out-patient basis and 12 completed physiotherapy. It was observed that more women had hemi-arthroplasty compared to men. The majority of the patients who had hemi-arthroplasty were aged seventy years and above and were not referred for full physiotherapy. There was poor documentation of the cause of fracture in the files of most of the cases reviewed, though a fall was the cause of fracture in the few that were documented. Outcome measures were underutilized with no record of reported patient-centred outcome measures for all the reviewed cases.
\end{abstract}

KEY WORDS: hemi-arthroplasty, full physiotherapy, ambulation

\section{INTRODUCTION}

Hemi-arthroplasty is a procedure in which the head and neck of the femur are replaced with a prosthesis, and the acetabulum is not modified (Bhattacharyya and Koval, 2009). Hemi-arthroplasty and total hip arthroplasty are often the treatment of choice after hip fracture, particularly among elderly people who have a displaced femoral neck fracture (Biber et al, 2012). These patients are typically frail and at high risk of complications and death (Rahme et al, 2010). In developing countries with limited health care facilities and with only a handful being capable of carrying out this procedure, coupled with the cost of the implants, the choice of arthroplasty becomes limited (Alonge and Shokumbi, 2004). Hemi-arthroplasty and total hip arthroplasty are commonly used to manage displaced intracapsular fractures of the femoral neck, each has disadvantages and the optimal treatment for these fractures is still subject to debate (Burgers et al, 2012). 
Femoral neck fractures, also known as intracapsular hip fractures, refer to fractures of the neck of the femur occurring within the capsule of the hip joint (Griffin, 2005). Femoral neck fracture occurs proximal to the point at which the hip joint capsule attaches to the femur (Parker, 2000). Femoral neck fractures can be further subdivided into those which are displaced (the fracture fragments are displaced relative to each other) and those which are essentially undisplaced.

Femoral neck fractures may occur at any age but are most common in elderly people (Beaupre et al, 2005). In the elderly population, these fractures are commonly seen after a trivial fall, and usually occur as a result of a fall from standing height. Poor bone density, multiple medical problems and the propensity to fall are major risk factors for femoral neck fracture. However, femoral neck fractures in adults younger than 50 years are uncommon and often the result of high-energy trauma. The mechanism of injury is often a motor vehicle accident or fall from a height. Fractures that occur in this normal bone density population require substantial axial load with the hip in an abducted position (Ly and Swiontkowski, 2008). They are associated with higher incidences of femoral head osteonecrosis and non-union (Dedrick et al, 1986; Upadhyay et al, 2004). The rate of osteonecrosis reported in the literature ranges from $12-86 \%$ in young patients after femoral neck fracture. This devastating complication may lead to collapse of the femoral head and subsequent osteoarthritis (Gautam et al, 1998).

In Africa, it appears that there are few published studies on femoral neck fractures. However, Ogunlade et al (2004) reported that femoral neck fracture is common in elderly patients but the incidence is less in Nigeria than in the Western world. In Nigeria, its incidence was reported to be 2.1 per 100,000 in men and 2.0 per 100,000 in women $\geq 50$ years (Ekezie et al, 2011). Some authors in Africa have documented incidences less than 90 per 100,000 people which is very low compared to findings for Europe and Asia (Ekezie et al, 2011; Dhanwal et al, 2011).

Physiotherapy after hip fracture is standard for most elderly patients, but there is a lack of consensus about the frequency and timing of therapy to maximize functional recovery after surgery (Bitar et al, 2005). Physiotherapy after hip replacement is accepted as a standard and essential treatment (Harper and Lyles, 1988). Its aim is to maximize functionality and independence and to minimize complications such as wound infection, deep vein thrombosis, pulmonary embolism, and hip dislocation (for hip replacement) (Bitar et al, 2005). Physiotherapy may be administered at several points following surgery, including immediately after (within the first 5 days) and in the early recovery period (within the first 3 months) after discharge (Handoll and Sherrington, 2007). There is a growing interest in whether physiotherapy should start before surgery. A variety of practices exist, and evidence regarding the optimal pre- and post-acute course of rehabilitation to obtain the best outcomes is needed (Jaglal et al, 2005). However, a search through the literature revealed that there is a dearth of studies on the clinical profile of patients with hip hemi-arthroplasty. This study was therefore designed to investigate the clinical profile of patients with hip hemi-arthroplasty managed at the physiotherapy department of a Nigerian tertiary health institution between 2008 and 2013.

\section{MATERIALS AND METHOD}

A 6-year review of cases of hip hemi-arthroplasty managed at the physiotherapy department of a tertiary institution in Nigeria was conducted. Permission to retrieve physiotherapy patients' case files for the study was obtained from the Head of Department of Physiotherapy. Clinical data of 57 patients who had hip hemi-arthroplasty seen at the physiotherapy department of the tertiary institution between 2008 and 2013 were retrieved from the physiotherapy register, physiotherapy patients' case files at the physiotherapy department record unit, referral case notes and the nursing register in the wards. A self-designed data form was used to document the following information: age, sex, cause of fracture, type of referral, discharge pattern, outcome assessed and treatment received. The research assistants were physiotherapists who assisted in retrieving the case files from the records section, and extracted required data from the case files.

\section{Data Analysis}

Descriptive statistics of frequency, mean and standard deviation were used to summarize age, while percentage was used to summarize sex, cause of fracture, types of referral, discharge pattern, outcome assessed and treatment received.

\section{RESULTS}

Fifty-seven case files of individuals referred to physiotherapy post hip hemi-arthroplasty were retrieved, 56 of which were patients who had hip hemi-arthroplasty secondary to femoral neck fracture while one case was due 
to femoral head damage secondary to sickle cell disease. Twenty-six $(45.6 \%)$ males and $31(54.4 \%)$ females with a mean age of $76.77 \pm 11.59$ years were recorded. The age range of the patients was 32-92 years with over $90 \%$ aged over 65 years (table 1). The cause of fracture was documented in only $21(36 \%)$ patients out of the 57 . The predominant cause of injury was falls in $17(77 \%)$ and road traffic accidents in $4(18 \%)$ of the patients. Only $16(28 \%)$ of the patients who underwent hip hemi-arthroplasty were referred for full physiotherapy, the remaining 41(72\%) were referred to physiotherapy for ambulation training only. Out of the 16 referred for physiotherapy, 14 continued physiotherapy on out-patient basis. None of the 41 patients referred for ambulation was seen as out-patients.

Table 1. Socio-demographic characteristics of the patients

\begin{tabular}{lcc}
\hline Variable & Frequency & Percentage \\
\hline Sex & 26 & \\
Male & 31 & 45.6 \\
Female & & 54.4 \\
Age Range & 4 & \\
$<65$ & 28 & 7 \\
$65-80$ & 25 & 49 \\
$>80$ & & 44 \\
Causes of Fracture & 4 & 7 \\
Road Traffic Accident & 17 & 29.82 \\
Fall & 1 & 1.75 \\
Avascular Necrosis of Head of Femur & 35 & 61.4 \\
Undocumented & & \\
\hline
\end{tabular}

Pain was assessed in all the $16(28 \%)$ patients referred for physiotherapy using the numerical rating scale (NRS) during initial examination. The patients had a mean pain intensity of $5.04 \pm 2.32$. Muscle strength was assessed in the $16(28 \%)$ patients using the Oxford muscle scale during the first assessment. There was no record of assessment of other health variables such as physical function, activities of daily living, quality of life, or functional ability, hence outcome measures on patient-centred outcomes were not used or were probably not documented.

A major proportion $(86 \%)$ of the patients were documented to have been discharged home using a Zimmer's frame as an assistive device for ambulation. Five $(8 \%)$ patients were discharged home on wheelchairs while the remaining three $(5 \%)$ died as in-patients; cause of deaths could not be ascertained in this review. Two (13\%) of the patients that continued as out-patients were discharged after they started ambulating independently while eleven $(69 \%)$ were discharged home using either a cane or a tetrapod. The remaining three (18\%) discontinued physiotherapy without discharge. There was emphasis on all necessary precautions in all $57(100 \%)$ patients to prevent dislocation of the prosthetic hip. In the physiotherapy management, there was emphasis on strengthening exercises to the affected limb in all $16(28 \%)$ patients who were referred for physiotherapy.

\section{DISCUSSION}

The aim of this study was to conduct a six-year review of cases of hip hemi-arthroplasty managed in the physiotherapy department of a Nigerian tertiary institution. The finding in this study that individuals who had hip hemiarthroplasty were aged 65 years and above and almost all sustained femoral fractures supports existing findings that hip hemi-arthroplasty is commonly done for elderly individuals who sustain femoral neck fractures (Biber et al, 2012; Beaupre et al, 2005; Fletcher et al, 2003). The results from this study also revealed that more females underwent hip hemi-arthroplasty as a result of femoral neck fracture. Tsai et al (2009) reported that femoral neck fracture was substantially greater in women than men.

The results from this study also show that the majority of patients who underwent hip hemi-arthroplasty were not referred for physiotherapy. This finding is a concern in the rehabilitation of patients who have had hemi-arthroplasty. Lowe et al (2009) reported that physiotherapy has long been a routine component of patient rehabilitation following hip joint replacement. Centre-based, but not home-based, exercise rehabilitation are effective in restoring muscle mass, strength and function in hip arthroplasty patients during the immediate post-surgery phase. The efficacy of the centre-based interventions is most likely due to higher training intensity that is facilitated by supervision and access to specialized equipment and facilities (Lemmey and Okoro, 2013).

The finding on discharge and referral pattern, in which the majority were simply referred for ambulation and not for in-patient physiotherapy, and discharged from the wards to outpatient physiotherapy is contrary to the findings of the Ontario Health Quality Report (2005) and Jae et al (2016). The authors in both studies reported that hip hemiarthroplasty patients, after discharge from an acute care hospital, may receive in-patient or out-patient physiotherapy as this will help to maximize functionality and independence. Jae et al (2016) reported that one month after hip surgery was the period for discharge home from the hospital or to complete the outpatient-based physical therapy. However, this was contrary to the findings of this study which revealed that the majority of the patients were 
discharged home just immediately after hip hemiarthroplasty without undergoing both in-patient and outpatient physiotherapy. It is necessary to state that it is inappropriate for patients who had hemi-arthroplasty to be referred for only ambulation and not the whole physiotherapy package. Standard treatment protocols for these individuals include restoring mobility, strength, flexibility and reducing pain (Stockton and Mergersen, 2009). It is hoped that the finding of non-referral of a large proportion of patients who underwent hemi-arthroplasty for physiotherapy will stimulate the development of guidelines and standard operating procedures in the management of these individuals.

The findings from this study reveal that ambulatory capacity of patients after hip hemi-arthroplasty varied. We observed that some of the patients ambulated independently while others needed assistive devices. The assistive devices used by the patients also differed; while some used canes or tetrapods, others ambulated with the use of wheelchairs. Jae et al (2016) reported that ambulatory capacity after hip fracture surgery can be divided into non-ambulatory state, ambulation with assistive device, and ambulation without assistive device. The study revealed that emphasis could have been placed on taking necessary precautions after hip hemi-arthroplasty in the management of these patients. This can be explained with the finding by Freburger (2000), who reported that the criteria for home discharge for patients referred for physical therapy frequently include the ability of the patient to physically demonstrate total hip precautions, verbally state total hip precautions, transfer independently, ambulate independently on level surfaces, and perform home exercise programme independently.

The findings that pain and muscle strength were the only impairment variables documented, as assessed in all the reviewed cases, can be explained by the fact that pain is a major symptom for patients' referral for physiotherapy. In the same vein, muscle strength assessment is central in musculoskeletal physiotherapy assessment (Steele et al, 2012). Monaghan et al (2012) reported that an eight-week exercise programme, with emphasis on strengthening and stability, resulted in statistically significant improvement in all measurements of self-perceived function and muscle strength in patients between four and twelve months post hip arthroplasty. It is however worthy of note that other impairment variables such as muscle atrophy and range of motion were not assessed. This finding is not consistent with the report of Holm et al (2013) that early strength deficits induced by surgery need to be quantified in order to design a specific, impairment-directed, postoperative rehabilitation programme for implementation soon after surgery. In addition, there was no documentation of the assessment of patient-centred outcomes such as walking, physical function, activities of daily living, quality of life, functional ability. This probably suggests that there should be a shift from healthcare providers' centred outcomes of impairments to patient-centered outcomes assessment. The finding from the review that the verbal rating scale was the only pain assessment tool utilized can be explained by the fact that it is a widely utilized tool in the geriatric population. It has been reported to be easily understood by the elderly who often report cognitive deficits when compared to the visual analogue scale (Krebs et al, 2007).

The finding that the predominant cause of femoral neck fractures in the documented cases was a fall supports the finding of Beaupre et al (2005) and Ly and Swiotkowski (2008) that these fractures are commonly seen after a trivial fall in the elderly population. Ly and Swiotkowski (2008) also reported poor bone density, multiple medical problems and propensity to fall as major risk factors for femoral neck fracture in the elderly. The finding that the cause of fracture was documented in about $36 \%$ of the cases reviewed is a pointer that there was incomplete documentation of patients' records. Thomas (2009) reported that it is important for healthcare providers to properly maintain the records of patients for two important reasons. The first one is that it will help them in the scientific evaluation of their patient profile, which is important for analysing treatment results and in planning treatment protocols. Second is that it helps in planning governmental strategies for future medical care. However, of equal importance in the present setting is in the issue of alleged medical negligence. The legal system relies mainly on documentary evidence in a situation where medical negligence is alleged by the patient or the relatives. Considering the introduction of the third party payers of health in the health care system in Nigeria, cases of litigation could come up as a result of poor or nondocumentation of patients' records.

Results from this study indicate that the physiotherapists formally discharged their patients as independent ambulation was attained during the study period. This finding is not in consonance with the finding by Odole et al (2013) where only $9 \%$ of cases of spinal pain reviewed were formally discharged. A plausible explanation for this could be that their review was conducted from 2006 to 2010 whilst that of this study was from 2008 to 2013. The difference in period of review could mean that 
physiotherapists have improved in their discharge practice appreciably. Odole et al (2013) advocated that there should be an audit of physiotherapy clinical practices as a means to ensuring that patients do not discontinue treatment of their own volition.

\section{CONCLUSION}

The results from this study revealed that more women had hemi-arthroplasty done compared with men. The majority of the patients who had hemi-arthroplasty were aged sixtyfive years and above. Also, the majority of the patients who had hip hemi-arthroplasty were not referred for physiotherapy. A fall was the cause of fracture in the majority of patients who had hip hemi-arthroplasty. There was poor documentation of the cause of fracture in the files of most of the cases reviewed. Outcome measures were underutilized with no record of reported patient-centred outcome measures for all the reviewed cases. There is the need for improvement in clinical documentation of cases and usage of standardized outcomes assessment in individuals who have hip hemi-arthroplasty managed at physiotherapy departments. This can be enhanced when treatment guidelines and protocols are developed by the regulatory body, physiotherapy departments and the orthopaedic manual therapy specialty group in Nigeria.

\section{REFERENCES}

Alonge T.O., Shokumbi W.A. 2004. The choice arthroplasty for secondary osteoarthritis of the hip joint following avascular necrosis of femoral head in sicklers. Journal of National Medical Association 96(5): 678-680.

Beaupre L.A., Cinats J.G., Senthilselvan A., Scharfenberger A., Johnston D.W., Saunders L.D. 2005. Does standardised rehabilitation and discharge planning improve functional recovery in elderly patients with hip fracture? Archives of Physical Medicine and Rehabilitation 86(12): 2231-2239.

Bhattacharyya T., Koval K.J. 2009. Unipolar versus bipolar hemiarthroplasty for femoral neck fractures: Is there a difference? Journal of Orthopeadics and Trauma 23(6): 426-427.

Biber R., Brem M., Singler K., Moeller M., Sieber C., Bail H.J. 2012. Dorsal versus transgluteal approach for hip hemiarthroplasty: An analysis of early complications in seven hundred and four consecutive cases. International Orthopaedics 36(11): 2219-2223.

Bitar A.A., Kaplan R.J., Stitik T.P., Shih V.C., Vo A.N., Kamen L.B. 2005. Rehabilitation of orthopaedic and rheumatologic disorder 3. Total hip arthroplasty rehabilitation. Archives of Physical Medicine and Rehabilitation 86(3): 556-560.

Burgers P.T.P., Van Greene A.R., Van den Bekerom M.P.J., Van Lieshout E.M.M., Blom B., Aleem I.S., Bhandari M., Poolman R.W. 2012. Total hip arthroplasty versus hemi- arthroplasty for displaced femoral neck fractures in healthy elderly: A meta-analysis and systematic review of randomised trials. International Orthopaedics 36(8): 1549-1560

Dedrick D.K., Mackenzie J.R., Burney R.E. 1986. Complications of femoral neck fracture in young adults. Journal of Trauma 6 : 932-7.

Dhanwal K.D., Dennison E.M.,Harvey N.C., Cooper C. 2011. Epidemiology of hip fracture: Worldwide geographic variation. Indian Journal of Orthopaedic 45(1): 15-22.

Ekezie J., Onwukamuche C.K., Anyawu G.E., Ugochuukwu A.I. 2011. Incidence of fall related hip fractures among the elderly persons in Owerri, Nigeria. Asian Journal of Medical Sciences 3(3): 110-114.

Fletcher A., Rigby A.S., Heyes T. 2003. Three-in-one femoral nerve block as analgesia for fracture neck of femur in the emmergency department: A randomised controlled trial. Annals of Emmergency Medicine 41: 227-233.

Freburger J.K. 2000. An analysis of relationship between the utilization of physical therapy services and outcome of care for patients after total hip arthroplasty. Physical Therapy 80(5): 448-458.

Gautam V.K., Anand S., Dhaon B.K. 1998. Management of displaced femoral neck fractures in young adults (a group at risk). Injury 29: 215-218.

Griffin L.Y. 2005. Essentials of Musculoskeletal Care. $3^{\text {rd }}$ ed Rosemont, IL: American Academy of Orthopaedic Surgeons.

Handoll H.H.G., Sherrington C. 2007. Mobilization strategies after hip fracture surgery in adults (Review). The Cochrane Collaboration, Issue $4 \mathrm{http} / / / \mathrm{www}$.thecochraneliberary. 12/10/2017, 9.38pm

Harper C.M., Lyles Y.M. 1988) Physiology and complications of bed rest. Journal of American Geriatric Society 36: 1047-1054.

Holm B., Thorborg K., Husted H., Kehlet H., Bandholm T. 2013. Surgery-induced changes and early recovery of hip-muscle strength, leg-press power, and functional performance after fast-track total hip arthroplasty: A prospective cohort study. Plos One. http://doi.org/10.1371/journal.pone.0062109

Jae L.M., Ji S.J., Sang J.K. 2016. Prediction of ambulatory status after hip fracture surgery in patients over 60 years old. Annals of Rehabilitation Medicine 40(4): 666-674.

Jaglal, S.B., Sherry, P.G., Schatzker, J. 2005. The impact and consequences of hip fracture in Ontario. Canadian Journal of Surgery 39: 105-111.

Krebs E.E., Carey T.S., Weinberger M. 2007. Accuracy of pain numeric rating scale as a screening test in primary care. Journal of General Internal Medicine 22(10): 1453-1458.

Lemmey A.B. and Okoro T. 2013. The efficacy of exercise rehabilitation in restoring physical function following total hip replacement for osteoarthritis: A review. OA Musculoskeletal Medicine 1: 1-20.

Lowe C.J., Barker K.L., Dewey M. E., Sackley C.M. 2009. Effectiveness of physiotherapy exercise following hip arthroplasty for osteoarthritis: a Systematic review of clinical trials. BMC Musculoskeletal Disorders 19(98): 1471-1474. 
Ly T.V., Swiontkowski M.F. 2008. Treatment of femoral neck fractures in young adults. Journal of Bones Joint Surgery 90(10): 2254-66.

Monaghan B., Grant T., Wayne H., Cusack T. 2012. Functional exercise after total hip replacement (FEATHER): A randomized clinical trial. BMC Musculoskeletal Disorder 13: 237.

Odole A.C., Ogunlana M.O., Akinpelu A.O., Oladejo K.S. 2013. Pattern of spinal pain management at the physiotherapy department of a tertiary health institution in Nigeria. African Journal of Physiotherapy and Rehabilitation Sciences 5(1\&2): 16-21.

Ogunlade S.O., Omololu A.B, Alonge T.O., Obajimi M.D. 2004. Femoral head diameter in subcapital fracture of the femur in Ibadan, Nigeria. African Journal of Medical Science 33(3): 235-238.

Ontario Health Quality Report. 2005. Physiotherapy rehabilitation after total knee or hip replacement. Ontario Health Technology Assessment Series 5(8).

Parker M.J. 2000. The management of intracapsular fractures of proximal femur. Journal of Bone Joint Surgery (Br) 82-B: $937-$ 941.
Rahme E., Kahn S.R., Dasgupta K., Burman M., Bernatsky S., Habel Y., Berry G. 2010. Short-term mortality associated with failure to receive home care after hemi-arthroplasty. Canadian Medical Association Journal 10: 1503/ Cmaj. 091209.

Steele L., Lade H., McKenzie S., Russell T.G. 2012. Assessment and diagnosis of musculoskeletal shoulder disorder over the internet. Internal Journal of Telemedicine. 2012:945745.

Stockson K.A., Mergersen K.A. 2009. Effects of multiple physiotherapy sessions on functional outcomes in the initial postoperative period after primary total hip replacement: a randomised control trial. Archieves of Physical Medicine and Rehabilitation (10): 1652-7.

Thomas J. 2009. Medical records and issues in negligence. Indian Journal of Urology 25(3): 384-388.

Tsai C., Hsu H., Fong Y., Lin C., Chen Y., Hsu C. 2009. Treatment of ilsilateral fractures of femoral neck and shaft. Injury 40(7): 778-782.

Upadhyay A., Jain P., Mishra P., Maini L., Gautum V.K., Dhaon B.K. 2004. Delayed internal fixation of fractures of the neck of femur in young adults. Journal of Bone Joint Surgery $(\mathrm{Br})$ 86: $1035-1040$. 